$\mathrm{ACT}-1 / 00$

CTP-TAMU-02/00

OUTP-00-03P

TPI-MINN-00/06

UMN-TH-1842-00

hep-ph/0002060

January 2000

\title{
Non-Abelian Flat Directions in a Minimal Superstring Standard Model
}

\author{
G.B. Cleaver, ${ }^{1,2}$ A.E. Faraggi, ${ }^{3}$ 地 D.V. Nanopoulos ${ }^{1,2,4}$, \\ and J.W. Walker ${ }^{1}$ f \\ ${ }^{1}$ Center for Theoretical Physics, Dept. of Physics, Texas A\&M University, \\ College Station, TX 77843, USA \\ ${ }^{2}$ Astro Particle Physics Group, Houston Advanced Research Center (HARC), \\ The Mitchell Campus, Woodlands, TX 77381, USA \\ ${ }^{3}$ Department of Physics, University of Minnesota, Minneapolis, MN 55455, USA, \\ and Theoretical Physics, University of Oxford, 1 Keble Road, Oxford OX1 3NP, UK \\ ${ }^{4}$ Academy of Athens, Chair of Theoretical Physics, Division of Natural Sciences, \\ 28 Panepistimiou Avenue, Athens 10679, Greece
}

\begin{abstract}
Recently, by studying exact flat directions of non-Abelian singlet fields, we demonstrated the existence of free fermionic heterotic-string models in which the $S U(3)_{C} \times S U(2)_{L} \times U(1)_{Y}$-charged matter spectrum, just below the string scale, consists solely of the MSSM spectrum. In this paper we generalize the analysis to include VEVs of non-Abelian fields. We find several, MSSM-producing, exact non-Abelian flat directions, which are the first such examples in the literature. We examine the possibility that hidden sector condensates lift the flat directions.
\end{abstract}

*gcleaver@rainbow.physics.tamu.edu

†faraggi@mnhepw.hep.umn.edu

$\ddagger$ dimitri@soda.physics.tamu.edu

§wwalker@rainbow.physics.tamu.edu 


\section{Minimal Superstring Standard Models}

The most realistic string models found to date [1, 2] have been constructed in the free fermionic formulation [3] of the heterotic-string. A large number of three generation models, which differ in their detailed phenomenological characteristics, have been built. All these models share an underlying $Z_{2} \times Z_{2}$ orbifold structure, which naturally gives rise to three generations with the $S O(10)$ embedding [4] of the Standard Model spectrumf. Recently, it was further demonstrated that free fermionic heterotic-string models can also produce models with solely the spectrum of the Minimal Supersymmetric Standard Model (MSSM) in the effective low energy field theory [5, 6, 7]. This is achieved due to the decoupling of all non-MSSM, exotic, and not-exotic, string states, at or slightly below the string scale, by Standard Model singlet VEVs which cancel the anomalous $U(1)$ D-term. This provides, for the first time, an example of a Minimal Standard Heterotic-String Model (MSHSM).

The emergence of a MSHSM in the free fermionic formulation reinforces the motivation for an improved understanding of this class of string compactifications. One of the important advancements of the last few years has been the development of techniques for systematic analysis of the $F$ - and $D$-flat directions of (string) models. Indeed, in demonstrating the existence of a free fermionic MSHSM we have utilized those improved techniques [5, 6, 7]. However, one limitation of those systematic studies performed to date is that they have included only flat directions of non-Abelian singlet fields. That is, fields which are singlets of all the non-Abelian gauge groups of a given string model and which may carry only Abelian $U(1)$ charges, or are singlets of the entire four dimensional gauge group. On the other hand, it has been shown in the past that some of the phenomenological constraints, such as quark-mixing [\&], may necessitate the use of non-Abelian VEVs. This was also suggested in our recent exploration of possible generational mass hierarchies and effective Higgs $\mu$ terms resulting from singlet VEVs in our MSHSM [7].

In this letter we therefore begin the task of extending the systematic analysis of flat directions for the cases which include non-Abelian VEVs. For our investigation we again start with the model we have denoted "FNY" first introduced in [1]. An important question that has been of some debate in previous studies, and is relevant for the question of supersymmetry breaking, is whether flat directions which include non-Abelian VEVs can be exact. Indeed, a particularly important result which we show here for the first time is the demonstration of a MSHSM solution which includes non-Abelian VEVs and is flat to all orders of nonrenormalizable terms. We further elaborate on the specific complications which arise in considering nonAbelian VEVs in the string models and briefly discuss some of the phenomenological implications of non-Abelian VEVs in the MSHSM. In a follow up to this letter 9 we will present a large collection of (systematically generated) non-Abelian MSHSM

* Among the three generation orbifold models, constructed to date, only the free fermionic models possess the $S O(10)$ embedding of the Standard Model spectrum 
$D$-flat directions that retain $F$-flatness to at least seventh order, along with a study of the phenomenological features of these directions.

\section{Non-Abelian Flat MSSM directions of the FNY model}

As advertised above, the model that we choose to study in this paper is the FNY model [1], which produced the first example of a MSHSM. The boundary conditions and GSO projection coefficients which define the model are given in ref. [1, 5, 6, 7] together with the cubic level and higher order terms in the superpotential. Here, we plunge directly to the analysis of the non-Abelian flat directions.

\section{$2.1 \quad$ Generic $D$ - and $F$-Flatness Constraints}

Spacetime supersymmetry is broken in a model when the expectation value of the scalar potential,

$$
V(\varphi)=\frac{1}{2} \sum_{\alpha} g_{\alpha} D_{a}^{\alpha} D_{a}^{\alpha}+\sum_{i}\left|F_{\varphi_{i}}\right|^{2}
$$

becomes non-zero. The $D$-term contributions in (2.1) have the form,

$$
D_{a}^{\alpha} \equiv \sum_{m} \varphi_{m}^{\dagger} T_{a}^{\alpha} \varphi_{m}
$$

with $T_{a}^{\alpha}$ a matrix generator of the gauge group $g_{\alpha}$ for the representation $\varphi_{m}$, while the $F$-term contributions are,

$$
F_{\Phi_{m}} \equiv \frac{\partial W}{\partial \Phi_{m}}
$$

The $\varphi_{m}$ are the scalar field superpartners of the chiral spin- $\frac{1}{2}$ fermions $\psi_{m}$, which together form a superfield $\Phi_{m}$. Since all of the $D$ and $F$ contributions to (2.1) are positive semidefinite, each must have a zero expectation value for supersymmetry to remain unbroken.

For an Abelian gauge group, the $D$-term (2.2) simplifies to

$$
D^{i} \equiv \sum_{m} Q_{m}^{(i)}\left|\varphi_{m}\right|^{2}
$$

where $Q_{m}^{(i)}$ is the $U(1)_{i}$ charge of $\varphi_{m}$. When an Abelian symmetry is anomalous, that is, the trace of its charge over the massless fields is non-zero,

$$
\operatorname{Tr} Q^{(A)} \neq 0
$$

the associated $D$-term acquires a Fayet-Iliopoulos (FI) term, $\epsilon \equiv \frac{g_{s}^{2} M_{P}^{2}}{192 \pi^{2}} \operatorname{Tr} Q^{(A)}$,

$$
D^{(A)} \equiv \sum_{m} Q_{m}^{(A)}\left|\varphi_{m}\right|^{2}+\epsilon
$$


$g_{s}$ is the string coupling and $M_{P}$ is the reduced Planck mass, $M_{P} \equiv M_{\text {Planck }} / \sqrt{8 \pi} \approx$ $2.4 \times 10^{18} \mathrm{GeV}$.

The FI term breaks supersymmetry near the string scale,

$$
V \sim g_{s}^{2} \epsilon^{2}
$$

unless its can be cancelled by a set of scalar VEVs, $\left\{\left\langle\varphi_{m^{\prime}}\right\rangle\right\}$, carrying anomalous charges $Q_{m^{\prime}}^{(A)}$,

$$
\left\langle D^{(A)}\right\rangle=\sum_{m^{\prime}} Q_{m^{\prime}}^{(A)}\left|\left\langle\varphi_{m^{\prime}}\right\rangle\right|^{2}+\epsilon=0
$$

To maintain supersymmetry, a set of anomaly-cancelling VEVs must simultaneously be $D$-flat for all additional Abelian and the non-Abelian gauge groups,

$$
\left\langle D^{i, \alpha}\right\rangle=0
$$

A non-trivial superpotential $W$ also imposes numerous constraints on allowed sets of anomaly-cancelling VEVs, through the $F$-terms in (2.1). F-flatness (and thereby supersymmetry) can be broken through an $n^{\text {th }}$-order $W$ term containing $\Phi_{m}$ when all of the additional fields in the term acquire VEVs,

$$
\left\langle F_{\Phi_{m}}\right\rangle \sim\left\langle\frac{\partial W}{\partial \Phi_{m}}\right\rangle \sim \lambda_{n}\langle\varphi\rangle^{2}\left(\frac{\langle\varphi\rangle}{M_{s t r}}\right)^{n-3}
$$

where $\varphi$ denotes a generic scalar VEV. If $\Phi_{m}$ additionally has a VEV, then supersymmetry can be broken simply by $\langle W\rangle \neq 0$. (The lower the order of an $F$-breaking term, the closer the supersymmetry breaking scale is to the string scale.)

\subsection{Non-Abelian flat directions in the FNY Model}

In [6] we classified the MSSM producing flat directions of the FNY model that are composed solely of singlet fields. Following this, in [0] we studied the phenomenological features of these singlet directions. We now consider here generalized MSSMproducing flat directions in the FNY model that contain non-Abelian VEVs. In our prior investigations we demanded stringent flatness. That is, we required $F$-flatness term by term in the superpotential, rather than allowing $F$-flatness to result from cancellation between terms. The absence of any non-zero terms from within $\left\langle F_{\Phi_{m}}\right\rangle$ and $\langle W\rangle$ is clearly sufficient to guarantee $F$-flatness along a given $D$-flat direction. However, such stringent demands are not necessary for $F$-flatness. Total absence of all individual non-zero VEV terms can be relaxed: collections of such terms appear without breaking $F$-flatness, so long as the terms separately cancel among themselves in each $\left\langle F_{\Phi_{m}}\right\rangle$ and in $\langle W\rangle$. However, even when supersymmetry is retained at a given order in the superpotential via cancellation between several terms in a specific $F_{\Phi_{m}}$, supersymmetry could well be broken at a slightly higher order. 
Non-Abelian VEVs offer one solution to the stringent $F$-flatness issue. Because non-Abelian fields contain more than one field component, self-cancellation of a dangerous $F$-term can sometimes occur along non-Abelian directions. That is, for some directions it may be possible to maintain "stringent" $F$-flatness even when dangerous $F$-breaking terms appear in the stringy superpotential. We will demonstrate selfcancellation of a non-Abelian direction using, as examples, the four non-Abelian flat directions, FDNA1 through FDNA4, presented in Table I. These four directions are the simplest MSSM $D$-flat non-Abelian directions that are also $F$-flat to at least seventh order. We will show that self-cancellation is not possible for FDNA1 and FDNA2, while it is for FDNA3 and FDNA4.

The singlet fields receiving VEVs, $\left\{\Phi_{12}, \Phi_{23}, \bar{\Phi}_{56}, \Phi_{4}, \Phi_{4}^{\prime}, \bar{\Phi}_{4}, \bar{\Phi}_{4}^{\prime}, H_{31}^{s}, H_{38}^{s}\right\}$, are the same for these four flat directions. The distinguishing aspect of these four directions is their non-Abelian components. The non-Abelian set for each direction is formed from a subset of the $S U(2)_{H}$ doublet fields $\left\{H_{23}, H_{26}, V_{40}\right\}$ and/or the $S U(2)_{H}^{\prime}$ doublet fields $\left\{H_{25}, H_{28}, V_{37}\right\}$. FDNA1 involves solely $S U(2)_{H}$ fields: $H_{23}$, $H_{26}$, and $V_{40}$, while FDNA2 is a $S U(2)_{H}^{\prime}$ parallel involving the corresponding $H_{25}$, $H_{28}$, and $V_{37}$. In contrast, FDNA3 and FDNA4 contain both $S U(2)_{H}$ and $S U(2)_{H}^{\prime}$ doublets: the sets $\left\{H_{23}, V_{40}, H_{28}, V_{37}\right\}$, and $\left\{H_{26}, V_{40}, H_{25}, V_{37}\right\}$, respectively.

Our four non-Abelian flat directions can be separated into two sets, \{FDNA1, FDNA2 $\}$ and $\{$ FDNA3, FDNA4 $\}$ due to a global $Z_{2}$ symmetry under which all $S U(2)_{H}$ and $S U(2)_{H}^{\prime}$ fields are exchanged: $H_{23} \leftrightarrow H_{25}, H_{26} \leftrightarrow H_{28}, V_{5} \leftrightarrow H_{9}, V_{7} \leftrightarrow$ $H_{10}, V_{15} \leftrightarrow H_{19}, V_{17} \leftrightarrow H_{20}, V_{25} \leftrightarrow H_{29}, V_{27} \leftrightarrow H_{30}, V_{39} \leftrightarrow H_{35}$, and $V_{40} \leftrightarrow H_{37}$. This symmetry is maintained in the superpotential to very high (and probably all) order in the superpotential. This implies that our findings regarding FDNA1 (FNDA3) have parallels for FDNA2 (FDNA4). Therefore we will examine only FDNA1 and FDNA3, but our findings will similarly apply to FDNA2 and FDNA4 after the appropriate field exchanges.

Now let us focus on FDNA1. In this $D$-flat direction the ratio of norms of the VEVs is:

$$
\begin{aligned}
\left|\left\langle\Phi_{12}\right\rangle\right|^{2} & =2\left|\left\langle\Phi_{23}\right\rangle\right|^{2}=\left|\left\langle\Phi_{56}\right\rangle\right|^{2}=\left|\left\langle H_{31}^{s}\right\rangle\right|^{2}=\left|\left\langle H_{38}^{s}\right\rangle\right|^{2} \\
& =2\left|\left\langle H_{23}\right\rangle\right|^{2}=2\left|\left\langle H_{26}\right\rangle\right|^{2}=\left|\left\langle V_{40}\right\rangle\right|^{2} \equiv 2|\langle\alpha\rangle|^{2} ; \quad \text { and } \\
\left(\left|\left\langle\Phi_{4}\right\rangle\right|^{2}\right. & \left.+\left|\left\langle\Phi_{4}^{\prime}\right\rangle\right|^{2}\right)-\left(\left|\left\langle\bar{\Phi}_{4}\right\rangle\right|^{2}+\left|\left\langle\bar{\Phi}_{4}^{\prime}\right\rangle\right|^{2}\right)=|\langle\alpha\rangle|^{2}
\end{aligned}
$$

where $\langle\alpha\rangle$ is the overall VEV scale determined by eq. (2.8),

$$
\langle\alpha\rangle=\sqrt{\frac{g_{s}^{2} M_{P}^{2} 1344 / 112}{192 \pi^{2}}} \approx 1 \times 10^{17} \mathrm{GeV} .
$$

This VEV ratio is fixed simply by the the Abelian $D$-terms (2.4,2.6) and the Cartan subalgebra (i.e., the diagonal) part of the $S U(2)_{H}$ and $S U(2)_{H}^{\prime} D$-terms.

\footnotetext{
${ }^{\dagger}$ For a list of the massless fields in the FNY model see [1], 6].
} 
In generic non-Abelian flat directions, the signs of the VEV components of a nonAbelian field are fixed by non-diagonal mixing of the VEVs in the corresponding nonAbelian $D$-terms (2.2). Since FDNA1 contains $S U(2)_{H}$ doublets, we must require

$$
\left\langle D^{S U(2)_{H}}\right\rangle=\left\langle H_{23}^{\dagger} T^{S U(2)} H_{23}+H_{26}^{\dagger} T^{S U(2)} H_{26}+V_{40}^{\dagger} T^{S U(2)} V_{40}\right\rangle=0,
$$

where

$$
T^{S U(2)} \equiv \sum_{a=1}^{3} T_{a}^{S U(2)}=\left(\begin{array}{cc}
1 & 1-i \\
1+i & -1
\end{array}\right)
$$

The only solutions to (2.15) consistent with $\left|\left\langle H_{23}\right\rangle\right|^{2}=\left|\left\langle H_{26}\right\rangle\right|^{2}=|\langle\alpha\rangle|^{2}$ are (up to a $\alpha \leftrightarrow-\alpha$ transformation)

$$
\left\langle H_{23}\right\rangle=\left(\begin{array}{r}
\alpha \\
-\alpha
\end{array}\right), \quad\left\langle H_{26}\right\rangle=\left(\begin{array}{r}
\alpha \\
-\alpha
\end{array}\right) \quad\left\langle V_{40}\right\rangle=\left(\begin{array}{r}
\sqrt{2} \alpha \\
\sqrt{2} \alpha
\end{array}\right),
$$

and

$$
\left\langle H_{23}\right\rangle=\left(\begin{array}{c}
\alpha \\
\alpha
\end{array}\right), \quad\left\langle H_{26}\right\rangle=\left(\begin{array}{c}
\alpha \\
\alpha
\end{array}\right) \quad\left\langle V_{40}\right\rangle=\left(\begin{array}{r}
\sqrt{2} \alpha \\
-\sqrt{2} \alpha
\end{array}\right) .
$$

A ninth-order superpotential term, $\Phi_{23} \bar{\Phi}_{56} \Phi_{4}^{\prime} H_{31}^{s} H_{38}^{s} H_{23} H_{26} V_{40} V_{39}$, jeopardizes the $F$-flatness of this non-Abelian $D$-flat direction via,

$$
\begin{aligned}
\left\langle F_{V_{39}}\right\rangle & \equiv\left\langle\frac{\partial W}{\partial V_{39}}\right\rangle \\
& \propto\left\langle\Phi_{23} \bar{\Phi}_{56} \Phi_{4}^{\prime} H_{31}^{s} H_{38}^{s}\right\rangle\left\langle H_{23} \cdot H_{26} V_{40}+H_{23} H_{26} \cdot V_{40}+H_{26} H_{23} \cdot V_{40}\right\rangle(2.2
\end{aligned}
$$

Self-cancellation of this F-term could occur if the non-Abelian VEVs resulted in

$$
\left\langle H_{23} \cdot H_{26} V_{40}+H_{23} H_{26} \cdot V_{40}+H_{26} H_{23} \cdot V_{40}\right\rangle=0 .
$$

However, neither (2.17) nor (2.18) produce this zero value. Instead, they generate

$$
\left\langle F_{V_{39}}\right\rangle=-\lambda_{9} \frac{8 \alpha^{8}}{M_{P}^{6}}\left(\begin{array}{l} 
\pm 1 \\
+1
\end{array}\right),
$$

with +1 for (2.17) and -1 for (2.18).

In contrast to FDNA1, we now show that self-cancellation of a dangerous $F$-term can occur for FDNA3. That is, non-Abelian $F$-term self-cancellation is consistent with $D$-term flatness for FDNA3. Along the FDNA3 direction, the ratio of the singlet VEVs is the same as for FDNA1 except for the $\Phi_{4}$ contribution. For FDNA3,

$$
\left(\left|\left\langle\Phi_{4}\right\rangle\right|^{2}+\left|\left\langle\Phi_{4}^{\prime}\right\rangle\right|^{2}\right)-\left(\left|\left\langle\Phi_{4}\right\rangle\right|^{2}+\left|\left\langle\bar{\Phi}_{4}^{\prime}\right\rangle\right|^{2}\right)=0 .
$$


The significant difference between FDNA3 and FDNA1 lies in FDNA3's non-Abelian VEV ratio,

$$
\left|\left\langle H_{23}\right\rangle\right|^{2}=\left|\left\langle V_{40}\right\rangle\right|^{2}=\left|\left\langle H_{28}\right\rangle\right|^{2}=\left|\left\langle V_{37}\right\rangle\right|^{2}=|\langle\alpha\rangle|^{2} .
$$

The $S U(2)_{H} D$-term,

$$
\left\langle D^{S U(2)_{H}}\right\rangle=\left\langle H_{23}^{\dagger} T^{S U(2)} H_{23}+V_{40}^{\dagger} T^{S U(2)} V_{40}\right\rangle=0
$$

has the two solutions

$$
\left\langle H_{23}\right\rangle=\left(\begin{array}{r}
\alpha \\
-\alpha
\end{array}\right), \quad\left\langle V_{40}\right\rangle=\left(\begin{array}{l}
\alpha \\
\alpha
\end{array}\right),
$$

and

$$
\left\langle H_{23}\right\rangle=\left(\begin{array}{l}
\alpha \\
\alpha
\end{array}\right), \quad\left\langle V_{40}\right\rangle=\left(\begin{array}{r}
\alpha \\
-\alpha
\end{array}\right) .
$$

The $S U(2)_{H}^{\prime} D$-term solutions for $H_{28}$ and $V_{37}$ have parallel form.

FDNA3's $F$-flatness is threatened by an eighth-order superpotential term,

$$
\Phi_{23} \bar{\Phi}_{56} H_{31}^{s} H_{38}^{s} H_{23} V_{40} H_{28} V_{35}
$$

through

$$
\begin{aligned}
\left\langle F_{V_{35}}\right\rangle & \equiv\left\langle\frac{\partial W}{\partial V_{35}}\right\rangle \\
& \propto\left\langle\Phi_{23} \bar{\Phi}_{56} H_{31}^{s} H_{38}^{s}\right\rangle\left\langle H_{23} \cdot V_{40}\right\rangle\left\langle H_{28}\right\rangle
\end{aligned}
$$

Either set of $S U(2)_{H} \operatorname{VEVs}(2.26)$ or (2.27) results in $\left\langle H_{23} \cdot V_{40}\right\rangle=0$. Hence $\left\langle F_{V_{35}}\right\rangle=0$ self-cancellation is consistent with $D$-flatness for FDNA3. Elimination of $\left\langle F_{V_{35}}\right\rangle$ makes FDNA3 flat to all finite order in the superpotential.

One can show that generic self-cancellation of a dangerous $F$-term occurs when, for at least one non-Abelian gauge group under which some of the flat direction VEVs carry charge, the ratio of the powers of the corresponding fields in the $F$-term is equivalent to the ratio of the norms of the flat direction VEVs carrying the given non-Abelian charge.

\section{Hidden Sector Condensates}

Non-Abelian VEV directions such as FDNA3 or FDNA4 can yield a three generation MSSM model while maintaining supersymmetry at the string /FI scale. Supersymmetry must ultimately be broken slightly above the electroweak scale, though. 
Along either of these two directions, our FNY model shows qualitatively how supersymmetry may be broken dynamically by hidden sector field condensation after either of these two directions is invoked. Recall that each of the flat directions FDNA3 and FDNA4 break both of the hidden sector $S U(2)_{H}$ and $S U(2)_{H}^{\prime}$ gauge symmetries, but leave untouched the hidden sector $S U(3)_{H}$. Thus, condensates of $S U(3)_{H}$ fields can initiate supersymmetry breaking [10].

The set of nontrivial $S U(3)_{H}$ fields is composed of five triplets,

$$
\left\{H_{42}, V_{4}, V_{14}, V_{24}, V_{34}\right\}
$$

and five corresponding anti-triplets,

$$
\left\{H_{35}, V_{3}, V_{13}, V_{23}, V_{24}\right\} \text {. }
$$

In both FDNA3 and FDNA4, singlet VEVs give unsuppressed FI-scale mass to two triplets, $V_{24}$ and $V_{34}$, and two anti-triplets, $V_{23}$ and $V_{33}$, via trilinear superpotential terms, fi

$$
\left\langle\Phi_{12}\right\rangle V_{23} V_{24}+\left\langle\Phi_{23}\right\rangle V_{33} V_{34}
$$

a slightly suppressed mass to one triplet/anti-triplet pair, $H_{42}$, and $H_{35}$, via a fifth order term,

$$
\left\langle\bar{\Phi}_{56} H_{31}^{s} H_{38}^{s}\right\rangle H_{42} H_{35}
$$

and a significantly suppressed mass to the pair, $V 4$ and $V 3$, via a tenth order term,

$$
\left\langle\Phi_{23} \bar{\Phi}_{56} H_{31}^{s} H_{38}^{s} H_{23} V_{40} H_{28} V_{37}\right\rangle V_{4} V_{3} .
$$

Before supersymmetry breaking, the last triplet/anti-triplet pair, $V_{14}$ and $V_{13}$, remain massless to all finite order.

Consider a generic $S U\left(N_{c}\right)$ gauge group containing $N_{f}$ flavors of matter states in vector-like pairings $T_{i} \bar{T}_{i}, i=1, \ldots N_{f}$. When $N_{f}<N_{c}$, the gauge coupling $g_{s}$, though weak at the string scale $M_{s t r}$, becomes strong at a condensation scale defined by

$$
\Lambda=M_{P} \mathrm{e}^{8 \pi^{2} / \beta g_{s}^{2}}
$$

where the $\beta$-function is given by,

$$
\beta=-3 N_{c}+N_{f}
$$

\footnotetext{
${ }^{\ddagger}$ Note that these $S U(3)_{H}$ triplet mass terms also occur along the simplest MSSM singlet flat direction possible [5, 6].
} 
The $N_{f}$ flavors counted are only those that ultimately receive masses $m \ll \Lambda$. Thus, for our model $N_{c}=3$ and $N_{f}=1$ (counting only the vector-pair, $V_{14}$ and $V_{13}$ ), which corresponds to $\beta=-8$ and results in an $S U(3)_{H}$ condensation scale

$$
\Lambda=\mathrm{e}^{-19.7} M_{P} \sim 7 \times 10^{9} \mathrm{GeV} .
$$

At this condensation scale $\Lambda$, the matter degrees of freedom are best described in terms of the composite "meson" fields, $T_{i} \bar{T}_{i}$. (Here the meson field is $V_{14} V_{13}$.) Minimizing the scalar potential of our meson field induces a VEV of magnitude,

$$
\left\langle V_{14} V_{13}\right\rangle=\Lambda^{3}\left(\frac{m}{\Lambda}\right)^{N_{f} / N_{c}} \frac{1}{m} .
$$

This results in an expectation value of

$$
\langle W\rangle=N_{c} \Lambda^{3}\left(\frac{m}{\Lambda}\right)^{N_{f} / N_{c}}
$$

for the non-perturbative superpotential.

Supergravity models are defined in terms of two functions, the Kähler function, $G=K+\ln |W|^{2}$, where $K$ is the Kähler potential and $W$ the superpotential, and the gauge kinetic function $f$. These functions determine the supergravity interactions and the soft-supersymmetry breaking parameters that arise after spontaneous breaking of supergravity, which is parameterized by the gravitino mass $m_{3 / 2}$. The gravitino mass appears as a function of $K$ and $W$,

$$
m_{3 / 2}=\left\langle\mathrm{e}^{K / 2} W\right\rangle
$$

Thus,

$$
m_{3 / 2} \sim\left\langle\mathrm{e}^{K / 2}\right\rangle\langle W\rangle \sim\left\langle\mathrm{e}^{K / 2}\right\rangle N_{c} \Lambda^{3}\left(\frac{m}{\Lambda}\right)^{N_{f} / N_{c}}
$$

Restoring proper mass units explicitly gives,

$$
m_{3 / 2} \sim\left\langle\mathrm{e}^{K / 2}\right\rangle N_{c}\left(\frac{\Lambda}{M_{P}}\right)^{3}\left(\frac{m}{\Lambda}\right)^{N_{f} / N_{c}} M_{P} .
$$

Our meson field $V_{14} V_{13}$ will acquire a mass of at least the supersymmetry breaking scale, so let us assume $m_{V_{14} V_{13}} \approx 1 \mathrm{TeV}$. The resulting gravitino mass is

$$
\begin{aligned}
m_{3 / 2} & \sim\left\langle\mathrm{e}^{K / 2}\right\rangle\left(\frac{7 \times 10^{9} \mathrm{GeV}}{2.4 \times 10^{18} \mathrm{GeV}}\right)^{3}\left(\frac{1000 \mathrm{GeV}}{7 \times 10^{9} \mathrm{GeV}}\right)^{1 / 3} 2.4 \times 10^{18} \mathrm{GeV} \\
& \approx\left\langle\mathrm{e}^{K / 2}\right\rangle 0.3 \mathrm{eV}
\end{aligned}
$$

In standard supergravity scenarios, one generally obtains soft-supergravitybreaking parameters, such as scalar and gaugino masses and scalar interaction, that 
are comparable to the gravitino mass: $m_{o}, m_{1 / 2}, A_{o} \sim m_{3 / 2}$. A gravitino mass of the order of the supersymmetry breaking scale would require $\left\langle\mathrm{e}^{K / 2}\right\rangle \sim 10^{12}$ or $\langle K\rangle \sim 55$. On the other hand, for a viable model, $\left\langle\mathrm{e}^{K / 2}\right\rangle \sim \mathcal{O}(1)$ would necessitate a decoupling of local supersymmetry breaking (parametrized by $m_{3 / 2}$ ) from global supersymmetry breaking (parametrized by $m_{o}, m_{1 / 2}$ ). This is indeed possible in the context of no-scale supergravity [11], endemic to weakly coupled string models.

In specific types of no-scale supergravity, the scalar mass $m_{o}$ and the scalar coupling $A_{o}$ have null values thanks to the associated form of the Kähler potential. Furthermore, the gaugino mass can go as a power of the gravitino mass, $m_{1 / 2} \sim\left(\frac{m_{3 / 2}}{M_{P}}\right)^{1-\frac{2}{3} q} M_{P}$, for the standard no-scale form of $G$ and a non-minimal gauge kinetic function $f \sim \mathrm{e}^{-A z^{q}}$, where $z$ is a hidden sector moduli field [12]. A gravitino mass in the range $10^{-5} \mathrm{eV} \lesssim m_{3 / 2} \lesssim 10^{3} \mathrm{eV}$ is consistent with the phenomenological requirement of $m_{1 / 2} \sim 100 \mathrm{GeV}$ for $\frac{3}{4} \gtrsim_{q} \gtrsim \frac{1}{2}$. Note that decoupling between the local and global breaking of supersymmetry also appears to be realized in strongly coupled heterotic strings [13].

\section{Discussion}

In this letter we have presented the four simplest non-Abelian $D$-flat directions of the FNY model that (i) produce exactly the MSSM fields as the only MSSMcharged fields in the low energy effective field theory and (ii) are flat to at least seventh order. F-flatness for the first two directions is necessarily broken by ninth order superpotential terms. For the last two directions, eighth order terms also pose a threat to $F$-flatness. All of these eighth and ninth order terms contain non-Abelian fields. For each of the latter two directions, we showed that a set of non-Abelian VEVs exist that is consistent with $D$-flat constraints and by which "self-cancellation" of the respective eighth order term can occur. By this, we mean that for each specific set of non-Abelian VEVs imposed by $D$-flatness constraints, the expectation value of the dangerous $F$-term is zero. Hence, the "dangerous" superpotential terms pose no problem and our latter two directions become flat to all finite order.

In [7] we discussed reasons why non-Abelian VEVs are likely required for a phenomenologically viable low energy effective MSSM, at least for the FNY string model. Evidence has also been presented in the past suggesting this might be true as well for all MSHSM $Z_{2} \times Z_{2}$ models. This implies that there is significant worth in exploring the generic properties of non-Abelian flat directions in $Z_{2} \times Z_{2}$ models that contain exactly the MSSM three generations and two Higgs doublets as the only MSSM-charged fields in the low energy effective field theory. For the next step in our study, we will present in [9] a large set of systematically generated MSSMproducing non-Abelian flat directions for the FNY model. We will then analyze the phenomenological differences between our non-Abelian directions and our past singlet directions. 


\section{Acknowledgments}

This work is supported in part by DOE Grants No. DE-FG-0294ER40823 (AF) and DE-FG-0395ER40917 (GC,DVN,JW). 


\section{A Example Non-Abelian $D$ - and $F$-flat MSSM Directions}

\begin{tabular}{|l||r||rrrrrr|rrr|rrr|}
\hline \hline FD \# & $Q^{\prime}$ & $\Phi_{12}$ & $\Phi_{23}$ & $\bar{\Phi}_{56}$ & $\left(\Phi_{4}\right)$ & $H_{31}^{s}$ & $H_{38}^{s}$ & $H_{23}$ & $H_{26}$ & $V_{40}$ & $H_{25}$ & $H_{28}$ & $V_{37}$ \\
\hline FDNA1 & -1 & 2 & 1 & 2 & 1 & 2 & 2 & 1 & 1 & 2 & & & \\
FDNA2 & -1 & 2 & 1 & 2 & 1 & 2 & 2 & & & & 1 & 1 & 2 \\
FDNA3 & -1 & 2 & 1 & 2 & 0 & 2 & 2 & 1 & & 1 & & 1 & 1 \\
FDNA4 & -1 & 2 & 1 & 2 & 0 & 2 & 2 & & 1 & 1 & 1 & & 1 \\
\hline \hline
\end{tabular}

Table I: Example FNY directions flat through at least seventh order that contain VEVs of Non-Abelian charged Hidden Sector Fields. All component VEVs in these directions are uncharged under the MSSM gauge group. Column one entries specify the class to which an example direction belongs. Column two entries give the anomalous charges $Q^{\prime} \equiv Q^{(A)} / 112$ of the flat directions. The next several column entries specify the ratios of the norms of the VEVs. The $\Phi_{4}-$ related component is the net value (in units of the square overall VEV scale) of $\left|\left\langle\Phi_{4}\right\rangle\right|^{2}+\left|\left\langle\Phi_{4}^{\prime}\right\rangle\right|^{2}-\left|\left\langle\bar{\Phi}_{4}\right\rangle\right|^{2}-\left|\left\langle\bar{\Phi}_{4}^{\prime}\right\rangle\right|^{2}$. E.g., a "1" in the $\Phi_{4}$ column for FDNA1 specifies that $\left|\left\langle\Phi_{4}\right\rangle\right|^{2}+\left|\left\langle\Phi_{4}^{\prime}\right\rangle\right|^{2}-\left|\left\langle\bar{\Phi}_{4}\right\rangle\right|^{2}-\left|\left\langle\bar{\Phi}_{4}^{\prime}\right\rangle\right|^{2}=1 \times|\langle\alpha\rangle|^{2}$.

\begin{tabular}{|l||l|l|}
\hline \hline FD\# & Dangerous $W$ Terms & Self-Cancellation $F$-Flatness Solution ? \\
\hline FDNA1 & $\Phi_{23} \bar{\Phi}_{56} \bar{\Phi}_{4} H_{31}^{s} H_{38}^{s} H_{23} H_{26} V_{40} V_{39}$ & No. \\
FDNA2 & $\Phi_{23} \bar{\Phi}_{56} \Phi_{4}^{\prime} H_{31}^{s} H_{38}^{s} H_{25} H_{28} V_{37} V_{35}$ & No. \\
FDNA3 & $\Phi_{23} \bar{\Phi}_{56} H_{31}^{s} H_{38}^{s} H_{23} V_{40} H_{28} V_{35}$ & Yes, via $\left\{\left\langle H_{23}\right\rangle,\left\langle V_{40}\right\rangle\right\}$. \\
FDNA4 & $\Phi_{23} \bar{\Phi}_{56} H_{31}^{s} H_{38}^{s} H_{26} V_{40} H_{25} V_{35}$ & Yes, via $\left\{\left\langle H_{26}\right\rangle,\left\langle V_{40}\right\rangle\right\}$. \\
\hline \hline
\end{tabular}

Table II: Dangerous F-breaking superpotential terms for flat directions in Table I. Column one entries specify the class of a flat direction. The entry in the next column specifies superpotential terms that can (possibly) break $F$-flatness and the last column entry indicates whether or not there is an allowed set of non-Abelian VEVs, consistent with $D$-flatness constraints, by which $F$-flatness may be maintained through self-cancellation. 


\section{References}

[1] A.E. Faraggi, D.V. Nanopoulos, and K. Yuan, Nucl. Phys. B335 (1990) 347;

A.E. Faraggi, Phys. Rev. D46 (1992) 3204.

[2] I. Antoniadis, J. Ellis, J. Hagelin, and D.V. Nanopoulos, Phys. Lett. B231 (1989) 65

J. Lopez, D.V. Nanopoulos and K. Yuan, Nucl. Phys. B399 (1993) 654;

I. Antoniadis. G.K. Leontaris and J. Rizos, Phys. Lett. B245 (1990) 161;

G.K. Leontaris, Phys. Lett. B372 (1996) 212;

G.K. Leontaris and J. Rizos, Nucl. Phys. B554 (1999) 3;

A.E. Faraggi, Phys. Lett. B274 (1992) 47; Phys. Lett. B278 (1992) 131; Nucl. Phys. B387 (1992) 239; Phys. Lett. B339 (1994) 223.

[3] I. Antoniadis, C. Bachas, and C. Kounnas, Nucl. Phys. B289 (1987) 87;

H. Kawai, D.C. Lewellen, and S.H.-H. Tye, Nucl. Phys. B288 (1987) 1.

[4] A.E. Faraggi and D.V. Nanopoulos, Phys. Rev. D48 (1993) 3288;

A.E. Faraggi, hep-th/9511093]; [hep-th/9708112].

[5] G.B. Cleaver, A.E. Faraggi and D.V. Nanopoulos, Phys. Lett. B455 (1999) 135, [hep-ph/9811427],

G.B. Cleaver, "M-Fluences on String Model Building," CTP-TAMU-46/98, hepph/9901203, Proceedings of CPT '98, November, 1998, Bloomington, Indiana.

[6] G.B. Cleaver, A.E. Faraggi and D.V. Nanopoulos, "A Minimal Superstring Standard Model I: Flat Directions," ACT-2/99, CTP-TAMU-12/99, TPI-MINN99/22, UMN-TH-1760-99, [hep-ph/9904301. To appear in Int. Jour. Mod. Phys. A.

[7] G.B. Cleaver, A.E. Faraggi, D.V. Nanopoulos, and J.W. Walker, "A Minimal Superstring Standard Model II: A Phenomenological Study," ACT-2/99, CTPTAMU-12/99, TPI-MINN-99/22, UMN-TH-1760-99, hep-ph/9910230.

[8] J. Lopez and D.V. Nanopoulos, Phys. Lett. B256 (1991) 150; ibid., B268 (1991) 359

I. Antoniadis, J. Rizos and K. Tamvakis, Phys. Lett. B278 (1992) 257;

A.E. Faraggi and E. Halyo, Nucl. Phys. B416 (1994) 63.

[9] G.B. Cleaver, A.E. Faraggi, D.V. Nanopoulos, and J.W. Walker, "Phenomenological Effects of Non-Abelian VEVs in a Minimal Superstring Standard Model," To appear.

[10] J.L. Lopez and D.V. Nanopoulos, Phys. Rev. Lett. 76 (1996) 1566, hep$\mathrm{ph} / 9511426$. 
[11] For a review see: A.B. Lahanas and D.V. Nanopoulos, Phys. Rep. 145 (1987) 1.

[12] J. Ellis, K. Enqvist, and D.V. Nanopoulos, Phys. Lett. B147 (1984) 99; Phys. Lett. B151 (1985) 357.

[13] P. Horava, Phys. Rev. D54 (1996) 7561, hep-th/9608019, and references therein. 